\title{
Stevens-Johnson syndrome associated with methotrexate treatment for acute lymphoblastic leukemia: a case report
}

\author{
Burcu Akıncı, M.D. ${ }^{a}$, Zuhal Ö. Siviş, M.D. ${ }^{a}$, Akkız Şahin, M.D. ${ }^{a}$, Deniz Y. Karapınar, M.D. ${ }^{a}$, \\ Can Balkan, M.D. ${ }^{a}$, Kaan Kavakl, M.D. ${ }^{a}$ and Yeşim Aydınok, M.D. ${ }^{a}$
}

\begin{abstract}
Toxic epidermal necrolysis and Stevens-Johnson syndrome are rare mucocutaneous diseases which are associated with a prolonged course and potentially lethal outcome. They are mostly drug induced and mortality rates are very high. Although mostly skin is involved, multiple organ systems such as cardiovascular, pulmonary, gastrointestinal, and urinary systems may be affected. Here, we report a case of StevensJohnson Syndrome associated with methotrexate treatment who developed acute cardiac failure and gastrointestinal hemorrhage beside skin findings. He had been treated with intravenous immunglobulin and methylprednisolone succesfully and continued chemotherapy with methotrexate treatment again.

Key words: Stevens-Johnson syndrome, toxic epidermal necrolysis, methotrexate, leukemia, child.
\end{abstract}

http: / / dx.doi.org/10.5546/ aap.2018.eng.e459

To cite: Akıncı B, Siviş ZO, Şahin A, et al. Stevens-Johnson syndrome associated with methotrexate treatment for acute lymphoblastic leukemia: a case report. Arch Argent Pediatr 2018;116(3):e459-e462.

\section{INTRODUCTION}

Toxic epidermal necrolysis (TEN) and StevensJohnson syndrome (SJS) are rare mucocutaneous diseases which are associated with a prolonged course and potentially lethal outcome. They are regarded as being two variants of the same process, differing solely in terms of the degree of surface area affected. They are typically drug induced. ${ }^{1}$ Mortality rate of SJS / TEN is very high ranging $1-35 \%$. Sepsis is the major cause of death. ${ }^{2}$ A severity of illness score (SCORTEN [Score of TEN]) has been devised to predict prognosis and expected mortality rate of these patients.

a. Ege University Faculty of Medicine, Department of Pediatrics, Division of Hematology, İzmir, Turkey.

E-mail address:

Burcu Akıncı, M.D.: bdeveci@windowslive.com

Funding: None.

Conflict of interest: None.

Received: 10-10-2017

Accepted: 11-28-2017
This scoring system addresses 7 prognostic risk factors. One point is assigned to each risk factor if positive and zero point if negative, with the total representing the final score. Table 1 shows the mortality risk which was anticipated according to the SCORTEN score. ${ }^{3}$ Treatment of SJS / TEN includes withdrawal of the offending drug, supportive care and "specific" drug treatment. ${ }^{4}$ Here, we report a case of StevensJohnson Syndrome associated with methotrexate treatment who had been treated succesfully and continued chemotherapy with methotrexate at the same dosage again.

\section{CASE REPORT}

The patient was an 8-year-old male who had been diagnosed with high risk acute lymphoblastic leukemia and enrolled on ALLIC BFM 2009 protocol which contains 6 High Risk (HR) blocks. Firstly, the patients get first HR1, HR2 and HR3 blocks. After that, they get the same blocks twice which are called as second HR1, HR2 and HR3. HR1 and HR2 blocks contain methotrexate at a dose of $5 \mathrm{~g} /$ $\mathrm{m}^{2}$. Our patient had completed first HR1 block in which he was exposed to methotrexate first

TABLE 1. The mortality risk which was anticipated according to the SCORTEN score

\begin{tabular}{lc}
\hline SCORTEN (Total score) & Mortality rate \\
\hline $0-1$ & $3.2 \%$ \\
2 & $12.2 \%$ \\
3 & $35.3 \%$ \\
4 & $58.3 \%$ \\
$\geq 5$ & $90 \%$ \\
Parameter & Score \\
Age $>40$ & No=0, Yes $=1$ \\
Total body surface area $>10 \%$ & $\mathrm{No}=0$, Yes $=1$ \\
Heart rate $>120$ beats per minute & $\mathrm{No}=0$, Yes $=1$ \\
Glucose level $>252 \mathrm{mg} / \mathrm{dl}$ & $\mathrm{No}=0$, Yes $=1$ \\
Serum urea level $>28 \mathrm{mg} / \mathrm{dl}$ & $\mathrm{No}=0$, Yes $=1$ \\
Bicarbonate level $<20 \mathrm{mEq} / \mathrm{L}$ & $\mathrm{No}=0$, Yes $=1$ \\
Presence of cancer $/$ & $\mathrm{No}=0$, Yes $=1$ \\
hematologic malignancy &
\end{tabular}


time without any complication. Then medullar punction was done and bone marrow was evaluated as in remission morphologically and first HR2 block was started. Two days after the end of block which he got methotrexate treatment second time, multiple maculopapuler lesions occurred on his legs and arms. Adequate intravenous hydration was started six hours before methotrexate treatment and continued to the last calcium folinate administration to provide good urine output. Also, intravenous alkalinization was done to maintain urine $\mathrm{pH}>7$. Before starting methotrexate infusion, his liver and renal function tests were completely normal. Plasma concentration of methotrexate was monitored carefully and determined in the normal range. Calcium folinate was administered at a dose of $15 \mathrm{mg} / \mathrm{m}^{2}$ at 42 hour, 48 hour and 54 hour after the start of methotrexate infusion. All of the medications which were incompetent with methotrexate such as trimethoprimsulfamethoxazole were withdrawn 24 hour before the infusion. Two days after the end of chemotherapy, the patient became neutropenic and he developed a fever. Over three days, atypical target lesions and red purpuritic macules covered approximately $10 \%$ of the body (Figure 1). The lesions then evolved into blisters resulting in sloughing of skin and positive Nikolsky's sign (Figure 2). Treatment involving replacement of fluid loss, maintaining electrolyte imbalance, topical medications and empiric broad spectrum

FIGURE 1. Atypical target lesions and red purpuritic macules

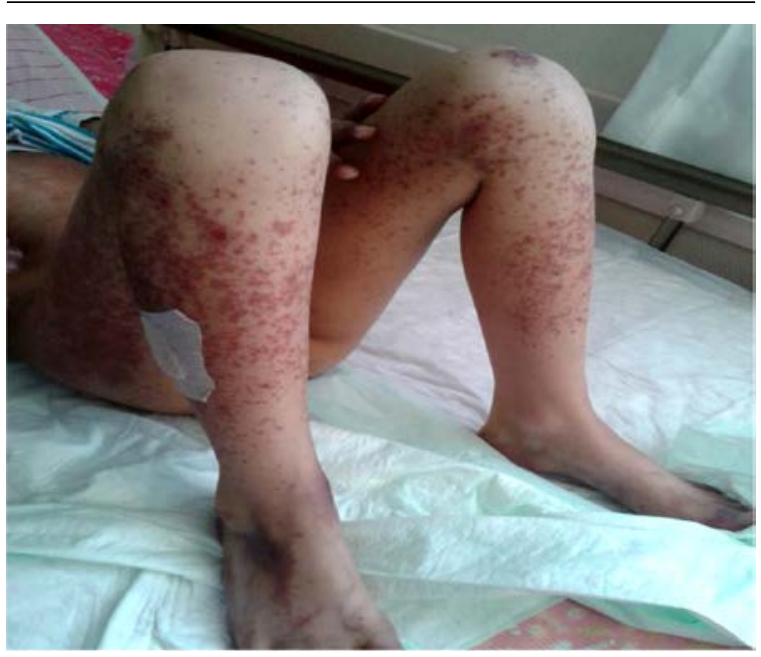

antibiotics was applied. Due to persistent fever on day 5 , computed chest tomography and abdomen ultrasonography were taken. Although there was no evidence of fungal infection, liposomal amphotericin B was added. During the course of several days, the patient's clinical status continued to deteriorate. He was tachycardic with a heart rate of $170 /$ minute and developed orthopnea. A transthoracic echocardiogram showed left ventricular dysfunction with an estimated left ventricular ejection fraction $45 \%$ showing cardiac failure. Digoxin, enalapril, carnitine and aldactazide were added for cardiac support. Also, he developed melena. He had edematous lips, oral and anal ulcerations (Figure 3). Packed red cell and platelet trasfusions were done. International normalized ratio and

FIGURE 2. Sloughing of skin

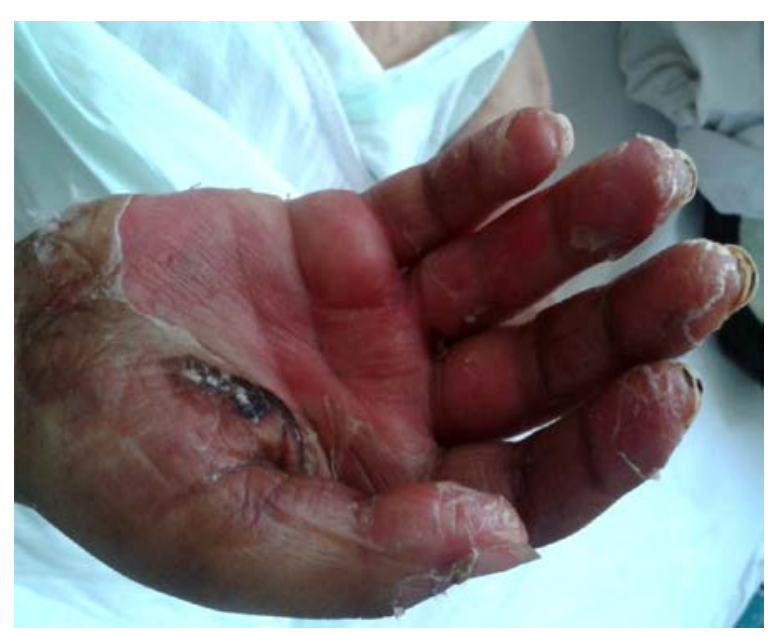

FIGURE 3. Oral ulceration

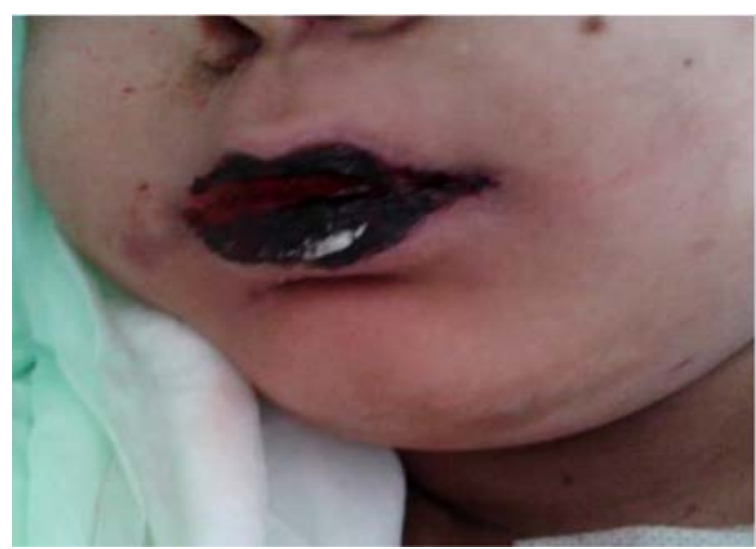


activated partial thromboplastin time was found as $1.5 \mathrm{sn}$ and 42 sn respectively; vitamin $\mathrm{K}$ was administered and fresh frozen plasma was given. The histopathologic examination of skin biopsy revealed focal parakeratosis on the surface, acanthosis in epidermis, vacuolar degeneration and individual cell necrosis in basal order, lymphocyte exocytosis and mild perivascular lymphocyte infiltration indicating interface changes which correlates with SJS / TEN. SCORTEN score was found as four. Intravenous immunglobulin at a dosage of $1 \mathrm{~g} / \mathrm{kg} /$ per day was given for three days. Also intravenous methylprednisolone at a dosage of $0.5 \mathrm{mg} / \mathrm{kg} /$ per day was administered for 7 days and gradually tappered off. Wound healing started at the end of the first week. After several days of antibiotic therapy and hemodynamic support, left ventricular ejection fraction improved progressively and the fever resolved. The patient exhibited a full recovery over the next 2 months and all drugs could be stopped. Just before the third administration of methotrexate, we prophylactically gave intravenous immunglobulin at a dosage of $1 \mathrm{~g} /$ $\mathrm{kg} /$ per day for three days. Approximately thirtysix hours later after the start of methotrexate treatment; hyperemia, rash and then bullae occurred at palms and soles of feet. We again gave intravenous immunglobulin for three consecutive days and started methylprednisolone at a dose of $2 \mathrm{mg} / \mathrm{kg} /$ day. Skin lesions did not progress and healing occurred within one week. Then steroid was tapered slowly. He did not have any problem in subsequent treatments. He underwent allogeneic hematopoetic stem cell transplantation and graft versus host disease did not occurred. The patient's follow-up still continues.

\section{DISCUSSION}

TEN and SJS are two rare but life-threatening mucocutaneous reactions characterized by acute skin blisters, scalding of the skin, and mucous membrane erosions. ${ }^{5,6}$ They are considered to be two ends of spectrum that detachment of $<10 \%$ of the total body surface area defines SJS, whereas $>30 \%$ defines TEN. Intermediate cases are called SJS/TEN overlap syndrome. ${ }^{2}$ The skin is often tender to the touch, with lateral pressure producing shedding of the epidermis from the dermis which is called as the Nikolsky's sign. ${ }^{1,7}$ Although mostly skin is involved, multiple organ systems such as cardiovascular, pulmonary, gastrointestinal, and urinary systems may be affected..$^{8-10}$ The patient in this case developed the full spectrum of the disease with involvement of all mucous membranes and skin involving less than $10 \%$ of the body surface area. Also acute heart failure and gastrointestinal hemorrhage occurred during the period of acute illness. He had a total of 4 positive factors (heart rate $>120$ / minute, serum urea $>28 \mathrm{mg} / \mathrm{dl}$, serum bicarbonate $<20 \mathrm{mEq} / \mathrm{L}$ and malignancy) according to the SCORTEN score corresponding to $58.3 \%$ mortality rate.

The pathophysiological mechanism is not fully understood, but it is believed that immune mediated hypersensitivity reaction is predominant. Due to death by apoptosis of keratinocytes, widespread epidermal necrosis occurs. The binding of Fas(CD95), a membrane receptor present in keratinocytes, with its FAS ligand (CD95L) lead to apoptosis of keratinocytes. ${ }^{1,11}$ Drug exposure is the cause of large majority of cases. The most commonly implicated drugs are antibiotics, non-steroidal anti-inflammatory drugs, and anticonvulsants. ${ }^{12,13}$ Methotrexate is an antifolate chemotherapeutic drug which is commonly used for treatment of acute lymphoblastic leukemia. The most common adverse effects of methotrexate are hepatotoxicity, ulcerative stomatitis, renal failure, and skin reactions.14 SJS-like exanthema in our patient was related to methotrexate administration and it occurred after second exposure of the drug which support immune mechanism of action. Diagnosis of SJS is based on clinical features and there is no universally accepted diagnostic criteria. Skin biopsy is not required routinely but histological features can support the diagnosis as in this case.

The management of SJS and TEN is mostly conservative. Treatment has three stages: withdrawal of the causative drug(s), rapidly initiating supportive care and ultimate"specific" drug therapy. Supportive care includes monitoring of fluids and electrolytes, barrier nursing care, nutritional support and control of infection. Randomized controlled studies have not been performed in specific treatment of SJS / TEN because they are rare and associated with a high rate of mortality. Intravenous immunglobulin may be an effective treatment by direct inhibition of FAS / FAS ligand interaction. 2,15 Also, corticosteroids has been proposed as a treatment strategy. The main mechanism of intended action is the modification of almost all components of the inflammatory and immune response. But the timing, corticosteroid type, 
dosage and duration remain unclear. ${ }^{3,15}$ Our case was treated with intravenous immunglobulin and steroid successfully. After healing period, we had to administer methotrexate again because of the risk group of patients. After third exposure to methotrexate, milder form of reaction occurred due to administration of intravenous immunglobulin and steroid before the infusion prophylactically.

In conclusion, SJS / TEN are rare clinical conditions with high mortality and morbidiy rate. Early diagnosis and administering appropriate treatment may improve the prognosis. Although the re-exposure of culprit drug after SJS / TEN is not recommended, if its usage is fundamental as in this case, it can be administered with intravenous immunglobulin and corticosteroid combination.

\section{REFERENCES}

1. Schwartz RA, McDonough PH, Lee BW. Toxic epidermal necrolysis: Part I. Introduction, history, classification, clinical features, systemic manifestations, etiology, and immunopathogenesis. J Am Acad Dermatol 2013;69(2):173. e1-13.

2. Harr T, French LE. Toxic epidermal necrolysis and StevenJohnson syndrome. Orphanet J Rare Dis 2010,5:39.

3. Bastuji-Garin S, Fouchard N, Bertocchi M, et al.SCORTEN: A severity-of-illness score for toxic epidermal necrolysis. J Invest Dermatol 2000;115(2):149-53.

4. Gerull R, Nelle M, Schaible T. Toxic epidermal necrolysis and Stevens-Johnson syndrome: A review. Crit Care Med 2011;39(6):1521-32.
5. Naldi L, CrottiS. Epidemiology of cutenous drug-induced reactions. G Ital Dermatol Venereal 2014;149(2):207-18.

6. French LE, Prins C. Toxic epidermal necrolysis. In: Bolognia JL, Jorizzo JL, Rapini RP, eds. Dermatology. Edinburgh: Mosby; 2003.P.323-31.

7. Grando SA, Grando AA, Glukhenky BT, et al. History and clinical significance of mechanical symptoms in blistering dermatoses: a reappraisal. J Am Acad Dermatol 2003;48(1): 86-92.

8. Hung CC, Liu WC, Kuo MC, et al. Acute renal failure and its risk factors in Stevens-Johnson syndrome and toxic epidermal necrolysis. Am J Nephrol 2009;29(6):633-8.

9. Lebargy F, Wolkenstein P, Gisselbrecht M, et al. Pulmonary complications in toxic epidermal necrolysis: a prospective clinical study. Intensive Care Med 1997;23(12):1237-44.

10. Michel P, Joly P, Ducrotte P, et al. Ileal involvement in toxic epidermal necrolysis (Lyell syndrome). Digest Dis Sci 1993;38(10):1938-41.

11. Wong A, Malvestiti AA, Silva Hafner MF. Stevens-Johnson syndrome and toxic epidermal necrolysis: a review. Rev Assoc Med Bras (1992). 2016;62(5):468-73.

12. Mockenhaupt M, Viboud C, Dunant A, et al. StevensJohnson syndrome and toxic epidermal necrolysis: assessment of medication risks with emphasis on recently marketed drugs. The Euro- SCAR-study. J Invest Dermatol 2008;128(1):35-44.

13. Levi N, Bastuji-GarinS, Mockenhaupt M, etal. Medications as risk factors of Stevens-Johnson syndrome and Toxic Epidermal Necrolysis in children: A pooled analysis. Pediatrics 2009;123(2):e297-304

14. Gaies E, Jebabli N, Trabelsi S, et al. Methotrexate side effects: review article. J Drug Metab Toxicol 2012,3:125.

15. Schwartz RA, McDonough PH, Lee BW. Toxic epidermal necrolysis: Part II. Prognosis, sequelae, diagnosis, differential diagnosis, prevention, and treatment. $J$ Am Acad Dermatol 2013;69(2):187.e1-16. 